\title{
Flux of Parallel Flow Momentum by Parallel Shear Flow Driven Instability
}

\author{
Yusuke KOSUGA ${ }^{1,2)}$, Sanae-I. ITOH ${ }^{2,3)}$ and Kimitaka ITOH ${ }^{3,4)}$ \\ 1) Institute for Advanced Study, Kyushu University, Fukuoka 812-8581, Japan \\ ${ }^{2)}$ Research Institute for Applied Mechanics, Kyushu University, Fukuoka 816-8580, Japan \\ ${ }^{3)}$ Research Center for Plasma Turbulence, Kyushu University, Fukuoka 816-8580, Japan \\ ${ }^{4)}$ National Institute for Fusion Science, Toki 509-5202, Japan
}

(Received 18 January 2016 / Accepted 29 January 2016)

\begin{abstract}
The flux of parallel momentum by parallel shear flow driven instability is calculated with the self-consistent mode dispersion. The result indicates that the diffusive component has two characteristic terms: $v_{D 1} \sim \tilde{v}_{x}^{2} / \gamma_{(0)}$ and $v_{D 2} \sim \tilde{v}_{x}^{2} /\left(k_{\|}^{2} D_{\|}\right)$where $\tilde{v}_{x}$ is the fluctuation radial velocity, $\gamma_{(0)}$ is the growth rate of the mode, $k_{\|}$is the parallel wave number, and $D_{\|}$is the electron diffusivity along the magnetic field. $v_{D 1}$ results when the parallel flow shear is above the threshold, while $v_{D 2}$ is important around the marginal state. Since typically $v_{D 1} \gg v_{D 2} \sim D_{n}$, where $D_{n}$ is the particle diffusivity, the Prandtl number $\left(\equiv v / D_{n}\right)$ becomes large when parallel flow shear driven instability occurs. This feature may explain the experimental observation on the difference between profiles of density and toroidal flow in edge and SOL plasmas.
\end{abstract}

(C) 2016 The Japan Society of Plasma Science and Nuclear Fusion Research

Keywords: parallel shear flow instability, dispersion relation, flux of parallel momentum

DOI: $10.1585 /$ pfr. 11.1203018

Flow along the magnetic field (parallel flow) is an important element to understand the behavior of magnetized plasmas. While parallel flows have beneficial effect for fusion plasmas by controlling transport, parallel flow shear itself can drive instability and be a source for fluctuation. The parallel flow shear driven instability was predicted by D'Agnelo [1], and the cases of NBI plasmas and SOL plasmas were analyzed [2-4]. It was shown that the Prandtl number is of order of unity in the case of drift waves [5]. More recently, the impact of parallel flow shear driven instability on particle transport and parallel momentum transport is reported from basic experiment [6], and analysis has been performed [7]. However, while the earlier studies reveal relevant contribution in transport fluxes and production rate, an approximate value for the frequency $\omega \sim \omega_{*}$, where $\omega_{*}$ is the drift wave frequency, was used. Though this is true for an order of magnitude estimate, the fluctuation of interest has a dispersion relation $\omega=\omega_{\mathbf{k}}$, and this has to be taken into account for more consistent analysis. Moreover, measurements of toroidal plasma flow near edge reported the difference between profiles of density and toroidal flow [8]. This motivates the theoretical evaluation of turbulent Prandtl number.

In this work, we present an analysis of transport of parallel flows by parallel flow shear driven instability, with the dispersion relation of the mode treated consistently. In order to address this, we use a fluid model [7] with the evolution of vorticity, density, and parallel flows:

author'se-mail: kosuga@riam.kyushu-u.ac.jp

$$
\begin{aligned}
& \frac{\mathrm{d}}{\mathrm{d} t} \rho_{\mathrm{s}}^{2} \nabla_{\perp}^{2} \frac{e \phi}{T_{\mathrm{e}}}=-D_{\|} \nabla_{\|}^{2}\left(\frac{e \phi}{T_{\mathrm{e}}}-\frac{n_{\mathrm{e}}}{n_{0}}\right), \\
& \frac{\mathrm{d}}{\mathrm{d} t} \frac{n_{\mathrm{e}}}{n_{0}}=-D_{\|} \nabla_{\|}^{2}\left(\frac{e \phi}{T_{\mathrm{e}}}-\frac{n_{\mathrm{e}}}{n_{0}}\right)-\nabla_{\|} v_{\mathrm{i}}, \\
& \frac{\mathrm{d}}{\mathrm{d} t} v_{\mathrm{i}}=-c_{\mathrm{s}}^{2} \nabla_{\|} \frac{n_{\mathrm{e}}}{n_{0}} .
\end{aligned}
$$

Here $\mathrm{d} / \mathrm{d} t=\partial_{\mathrm{t}}+(c / B) \hat{z} \times \nabla \phi, \phi$ is the electrostatic potential, $\rho_{\mathrm{s}}$ is the ion sound Larmor radius, $D_{\|}=v_{\text {the }}^{2} / v_{\mathrm{e}}$ is the parallel diffusivity of electrons, $v_{\mathrm{e}}$ is the electron collision frequency, $n_{\mathrm{e}}$ is the electron density, $n_{0}$ is a reference density, $v_{\mathrm{i}}$ is the ion fluid velocity, $c_{\mathrm{s}}$ is the ion sound speed.

Linearization of the model equation yields the dispersion relation as:

$$
\frac{\rho_{\mathrm{s}}^{2} k_{\perp}^{2}}{i k_{\|}^{2} D_{\|}} \omega=\frac{-\left(\omega-\omega_{* \mathrm{e}}\right) \omega+\left(c_{\mathrm{s}}^{2} k_{\|}^{2}-c_{\mathrm{s}} k_{\|} \rho_{\mathrm{s}} k_{y}\left\langle v_{\|}\right\rangle^{\prime}\right)}{\left(\omega+i k_{\|}^{2} D_{\|}\right) \omega-c_{\mathrm{s}}^{2} k_{\|}^{2}} .
$$

Here $\omega_{* \mathrm{e}}=k_{y}\left(\rho_{\mathrm{s}} / L_{n}\right) c_{\mathrm{s}}, k_{\perp}^{2}=k_{x}^{2}+k_{y}^{2}$, and $L_{n}^{-1}=-\langle n\rangle^{\prime} / n_{0}$ is the density scale length. Equation (4) is solved perturbatively using the inverse of the adiabaticity parameter $\omega_{* \mathrm{e}} /\left(k_{\|}^{2} D_{\|}\right) \ll 1$. For parallel flow shear driven mode, the dispersion relation is:

$$
\begin{aligned}
& \omega_{r,(0)}=\frac{\omega_{* \mathrm{e}}}{2\left(1+k_{\perp}^{2} \rho_{\mathrm{s}}^{2}\right)}, \\
& \gamma_{(0)}=\operatorname{sgn}\left(k_{y}\right) \frac{\omega_{* \mathrm{e}}}{2\left(1+k_{\perp}^{2} \rho_{\mathrm{s}}^{2}\right)} \sqrt{\mathcal{D}_{v}}, \\
& \mathcal{D}_{v}=4\left(1+\rho_{\mathrm{s}}^{2} k_{\perp}^{2}\right)\left(\frac{k_{\|} L_{n}}{k_{y} \rho_{\mathrm{s}}} \frac{\left\langle v_{z}\right\rangle^{\prime}}{c_{\mathrm{s}} / L_{n}}-\frac{k_{\|}^{2} L_{n}^{2}}{k_{y}^{2} \rho_{\mathrm{s}}^{2}}\right)-1 .
\end{aligned}
$$


Basic feature of the instability is obtained from the zeroth order growth rate and is reported in literature [7,9]. Here note that the onset of the instability requires parallel flow shear needs to be large enough to make $\mathcal{D}_{v}>0$. We also note that the parallel wave number should not be too large to avoid the stabilizing effect due to acoustic wave coupling. The adiabaticity condition $\omega_{* \mathrm{e}} /\left(k_{\|}^{2} D_{\|}\right) \ll 1$ is guaranteed with the large parallel electron diffusivity. The next order correction is calculated as

$$
\begin{aligned}
& \delta \omega_{r}=\operatorname{sgn}\left(k_{y}\right) \frac{\omega_{* \mathrm{e}}^{2}}{k_{\|}^{2} D_{\|}^{2}} \frac{1}{\sqrt{\mathcal{D}_{v}}} \frac{k_{\perp}^{2} \rho_{\mathrm{s}}^{2}}{2\left(1+k_{\perp}^{2} \rho_{\mathrm{s}}^{2}\right)^{2}} \\
& \times\left\{\frac{1}{1+k_{\perp}^{2} \rho_{\mathrm{s}}^{2}}-3 \frac{k_{\|} L_{n}}{k_{y} \rho_{\mathrm{s}}} \frac{\left\langle v_{\|}\right\rangle^{\prime}}{c_{\mathrm{s}} / L_{n}}+\left(2-k_{\perp}^{2} \rho_{\mathrm{s}}^{2}\right) \frac{k_{\|}^{2} L_{n}^{2}}{k_{y}^{2} \rho_{\mathrm{s}}^{2}}\right\} \\
& \delta \gamma=\frac{\omega_{* \mathrm{e}}^{2}}{k_{\|}^{2} D_{\|}^{2}} \frac{k_{\perp}^{2} \rho_{\mathrm{s}}^{2}}{2\left(1+k_{\perp}^{2} \rho_{\mathrm{s}}^{2}\right)^{2}} \\
& \times\left\{\frac{1}{1+k_{\perp}^{2} \rho_{\mathrm{s}}^{2}}-\frac{k_{\|} L_{n}}{k_{y} \rho_{\mathrm{s}}} \frac{\left\langle v_{\|}\right\rangle^{\prime}}{c_{\mathrm{s}} / L_{n}}-k_{\perp}^{2} \rho_{\mathrm{s}}^{2} \frac{k_{\|}^{2} L_{n}^{2}}{k_{y}^{2} \rho_{\mathrm{s}}^{2}}\right\}
\end{aligned}
$$

The flux of parallel momentum is calculated. General expression is obtained by using the quasilinear theory [10]

$$
\begin{aligned}
& \frac{\Pi_{x \|}}{c_{\mathrm{s}}^{2}}=\operatorname{Re} \sum_{\mathbf{k}} k_{y} \rho_{\mathrm{s}} \frac{\left(-\omega+\omega_{* \mathrm{e}}\right) c_{\mathrm{s}} k_{\|}}{k_{\|}^{2} D_{\|} \omega}\left|\frac{e \tilde{\phi}_{\mathbf{k}}}{T_{\mathrm{e}}}\right|^{2} \\
& +\operatorname{Re} \sum_{\mathbf{k}} i k_{y} \rho_{\mathrm{s}} \frac{c_{\mathrm{s}} k_{\|}-\rho_{\mathrm{s}} k_{y}\left\langle v_{\|}\right\rangle^{\prime}}{\omega}\left(1-i \frac{k_{\|}^{2} c_{\mathrm{s}}^{2}}{k_{\|}^{2} D_{\|} \omega}\right)\left|\frac{e \tilde{\phi}_{\mathbf{k}}}{T_{\mathrm{e}}}\right|^{2} .
\end{aligned}
$$

Using the dispersion relations Eqs. (5), (6), (8) and (9), the momentum flux reduces to

$$
\begin{aligned}
\frac{\Pi_{x \|}}{c_{\mathrm{s}}^{2}}= & -\sum_{\mathbf{k}} \frac{k_{y} \rho_{\mathrm{s}}}{c_{\mathrm{s}} k_{\|}} \gamma_{(0)}\left(1+k_{\perp}^{2} \rho_{\mathrm{s}}^{2}\right)\left|\frac{e \tilde{\phi}_{\mathbf{k}}}{T_{\mathrm{e}}}\right|^{2} \\
& -\sum_{\mathbf{k}} \frac{k_{y}^{2} \rho_{\mathrm{s}}^{2}}{k_{\|}^{2} D_{\|}} \frac{k_{\perp}^{2} \rho_{\mathrm{s}}^{2}}{2\left(1+k_{\perp}^{2} \rho_{\mathrm{s}}^{2}\right)}\left|\frac{e \tilde{\phi}_{\mathbf{k}}}{T_{\mathrm{e}}}\right|^{2}\left\langle v_{\|}\right\rangle^{\prime} \\
& +\sum_{\mathbf{k}} \frac{k_{y} \rho_{\mathrm{s}} c_{\mathrm{s}} k_{\|}}{k_{\|}^{2} D_{\|}} \frac{k_{\perp}^{2} \rho_{\mathrm{s}}^{2}\left(2+k_{\perp}^{2} \rho_{\mathrm{s}}^{2}\right)}{2\left(1+k_{\perp}^{2} \rho_{\mathrm{s}}^{2}\right)}\left|\frac{e \tilde{\phi}_{\mathbf{k}}}{T_{\mathrm{e}}}\right|^{2}
\end{aligned}
$$

Note that in the adiabatic limit, only the first term remains finite. When parallel flow shear exceeds the critical value, this term is approximately given as

$$
\begin{aligned}
& -\sum_{\mathbf{k}} \frac{k_{y} \rho_{\mathrm{s}}}{c_{\mathrm{s}} k_{\|}} \frac{\gamma_{(0)}^{2}}{\gamma_{(0)}}\left(1+k_{\perp}^{2} \rho_{\mathrm{s}}^{2}\right)\left|\frac{e \tilde{\phi}_{\mathbf{k}}}{T_{\mathrm{e}}}\right|^{2} \\
& \cong-\sum_{\mathbf{k}} \frac{k_{y}^{2} \rho_{\mathrm{s}}^{2}}{\gamma_{(0)}}\left|\frac{e \tilde{\phi}_{\mathbf{k}}}{T_{\mathrm{e}}}\right|^{2}\left\langle v_{\|}\right\rangle^{\prime} .
\end{aligned}
$$

This is diagonal, diffusive flux $-v_{D 1}\left\langle v_{\|}\right\rangle^{\prime}$ with the viscosity given by

$$
v_{D 1} \sim \frac{\tilde{v}_{x}^{2}}{\gamma_{(0)}} .
$$

The second term in the momentum flux is also related to the diffusive component, with the viscosity

$$
v_{D 2} \sim \frac{\tilde{v}_{x}^{2}}{k_{\|}^{2} D_{\|}} .
$$

There may be a competing effect from the residual stress, the last term in the momentum flux.

The viscosity induced by the parallel flow shear driven instability has two characteristic values, $v_{D 1}$ and $v_{D 2}$. The difference in the viscosity may be used to apply parallel flow shear driven instability to experiment. Since typically $k_{\|}^{2} D_{\|} \gg \omega_{*} \sim \gamma$ holds, we have $v_{D 1} \gg v_{D 2}$. Thus, once the parallel flow velocity exceeds the critical value, parallel flow shear driven modes appear and induce the large viscosity $v_{D 1}$ to relax the parallel flow profile. Around the marginal state, the first term of the momentum flux is small, and the profile relaxes due to $v_{D 2}$. Defining the Prandtl number $\operatorname{Pr} \equiv v_{D} / D_{n}$ with the particle diffusivity $D_{n} \sim \tilde{v}_{x}^{2} /\left(k_{\|}^{2} D_{\|}\right)$, this feature is summarized as $\operatorname{Pr} \gg 1$ when parallel flow shear driven instability appears, while $\mathrm{Pr} \sim O(1)$ when the parallel flow shear is around the critical value. The large Prandtl number can induce a broader profile of toroidal flow than that of density profile. This can be one of the reasons for the broader profile of toroidal flow in SOL plasmas, which was reported in [8]. In addition, variation of Prandtl number might introduce a new mechanism in profile formation near edge and SOL, as has been theoretically pointed out in conjunction with the density peaking phenomena [11].

In summary, we presented an analysis of the flux of parallel momentum driven by parallel flow shear driven instability, with the mode dispersion treated consistently. The results indicate that the viscosity on the flow has two typical values. Above the critical velocity shear, the viscosity is larger than the particle diffusivity, so the Prandtl number is larger than one. On the other hand, if the shear is close to the marginal, the Prandtl number is $\sim O(1)$. These features may be important to understand the difference in the profile structures of toroidal flow and density reported from experiments.

We thank Drs. S. Inagaki, T. Kobayashi, P.H. Diamond, Ö.D. Gürcan, and the participants in the Festival de Theorie for stimulating discussions. This work is partly supported by the Grants-in-Aid for Scientific Research of JSPS of Japan (23244113, 15K17799, 15H02155), Asada Science Foundation, Kyushu University Interdisciplinary Programs in Education and Projects in Research Development (26705), and the Collaborative Research Program of Research Institute for Applied Mechanics, Kyushu University.

[1] N. D’Angelo, Phys. Fluids 8, 1748 (1965).

[2] P.J. Catto, M.N. Rosenbluth and C.S. Liu, Phys. Fluids 16, 1719 (1973).

[3] N. Mattor and P.H. Diamond, Phys. Fluids 31, 1180 (1988). 
[4] X. Garbet, C. Fenzi, H. Capes, P. Devynck and G. Antar, Phys. Plasmas 6, 3955 (1999).

[5] S.-I. Itoh, Phys. Fluids B 4, 796 (1992).

[6] S. Inagaki et al., accepted to Sci. Rep. (2016).

[7] Y. Kosuga, S.-I. Itoh and K. Itoh, Plasma Fusion Res. 10, 3401024 (2015).

[8] B. LaBombard et al., Nucl. Fusion 44, 1047 (2004).
[9] Y. Kosuga, S.-I. Itoh and K. Itoh, accepted to Contrib. Plasma Phys. (2016).

[10] P.H. Diamond, S.-I. Itoh and K. Itoh, Modern Plasma Physics Vol.1: Physical Kinetics of Turbulent Plasmas (Cambridge University Press, Cambridge, 2011).

[11] S.-I. Itoh, K. Itoh and A. Fukuyama, J. Nucl. Mater. 220222, 117 (1995). 